\title{
Ausencia congénita de incisivos laterales superiores. Enfoques terapéuticos y sus resultados a largo plazo. Revisión descriptiva.
}

\section{Congenital absence of upper lateral incisors. Therapeutic approaches and their long-term results. Descriptive review.}

\begin{abstract}
Carlos Vega ${ }^{\star}$, Josefina Pinto ${ }^{2}$, Tamara Tejeda ${ }^{2}$, Victor Rojas ${ }^{1}$
1. Departamento de Ortodoncia, Facultad de Odontología Universidad de los Andes. Santiago, Chile.

2. Alumna de Odontología, Facultad de Odontología Universidad de los Andes. Santiago, Chile.

* Correspondencia Autor: Carlos Vega | Dirección: Monseñor Álvaro del Portillo 12.455, Las Condes, Santiago, Chile. | Teléfono: +56994499026 | E-Mail: cvegap@gmail.com

Trabajo recibido el 28/03/2021

Trabajo revisado 13/06/2021

Aprobado para su publicación el 10/07/2021

RESUMEN

La agenesia de incisivos laterales superiores corresponde a un desafío clínico, en cuanto a planificación de tratamiento, así como también para la obtención de resultados estéticos y funcionales adecuados. Con el fin de conseguirlo, el clínico debería informarse sobre las diferentes opciones terapéuticas disponibles en la actualidad, considerando que la indicación depende de cada caso clínico individual, es decir, del paciente en su contexto integral sumado a su condición intra-oral. Además, es importante evaluar los resultados a largo plazo de las diferentes alternativas, para lograr la resolución clínica más adecuada y estable en el tiempo. El éxito en estos pacientes, requiere de un enfoque multidisciplinario, considerando especialistas en ortodoncia, rehabilitación oral, periodoncia y cirugía oral de ser necesario. El objetivo de esta revisión es describir y comparar las alternativas terapéuticas para la agenesia de incisivos laterales maxilares.

PALABRAS CLAVE:
\end{abstract}

ORCID

Victor Rojas: 0000-0001-7653-8681

Carlos Vega: 0000-0002-8081-5925

Agenesia; Incisivos laterales; Tratamiento; Ortodoncia; Implantes.

Int. J. Inter. Dent Vol. 14(3); 257-265, 2021.

\section{ABSTRACT}

The agenesis of upper lateral incisors represents a clinical challenge regarding treatment planning and appropriate aesthetic and functional results. In order to obtain the best results, the clinician should be informed about the different therapeutic options currently available, considering that the indication depends on each individual clinical case, that is, on the patient in his/her comprehensive context in addition to his/her oral condition. It is also important to evaluate long term results from each treatment available, in order to achieve the better outcome and most importantly, a stable clinical resolution over time. The treatment success in this kind of patients requires a multidisciplinary approach, including specialists in orthodontics, oral rehabilitation, periodontics, and oral surgery if necessary. The objective of this review is to describe and compare the therapeutic alternatives for maxillary lateral incisor agenesis.

KEY WORDS:

Agenesis; Lateral incisors; Treatment; Orthodontics; Implants.

Int. J. Inter. Dent Vol. 14(3); 257-265, 2021.

\section{INTRODUCCIÓN}

La agenesia de incisivos laterales superiores corresponde a la más común de las ausencias dentarias congénitas, después de los terceros molares. Trae consigo un compromiso estético y funcional, que necesita de un manejo multidisciplinario(1). La prevalencia corresponde a un 0.8$2 \%$ de la población en general ${ }^{(2)}$.

Considerando que el diagnóstico y el tratamiento suele ser en edad de crecimiento y desarrollo craneofacial, las alternativas terapéuticas para el reemplazo o sustitución de los dientes ausentes siguen aún en controversia( ${ }^{(3)}$.

Dentro de las alternativas terapéuticas para la sustitución de estos dientes, está el cierre de espacios mediante ortodoncia, con el reemplazo del incisivo lateral faltante por el canino adyacente; apertura de espacio para la colocación de un implante óseo integrado y su posterior rehabilitación; autotrasplante dentario y restauración con pónticos adheridos a las piezas dentarias adyacentes ${ }^{(4)}$. Cada una de estas alternativas busca éxito a largo plazo, junto con estética y función aceptable(5).

El reemplazo del incisivo lateral faltante por el canino adyacente mediante movimientos de ortodoncia, pareciera ser la opción más conservadora, con buenos resultados y estabilidad a largo plazo ${ }^{(2,6)}$. Sin embargo, requiere de restauraciones que desgastan estructura dental sana, con el fin de recuperar la armonía y estética del arco dentario(2).

En cuanto a la apertura de espacios y posterior instalación de implantes oseointegrados, es necesario tener en cuenta la edad del paciente, debido al riesgo de infraoclusión, sin embargo, se habla de que la aparición de esta es independiente de la edad e inevitable. Esto se explica debido a que el implante es incapaz de seguir la dirección y velocidad de crecimiento dentoalveolar de las piezas dentarias adyacentes. Además, se han observado alteraciones en relación a los tejidos blandos, comprometiendo la estética de la restauración ${ }^{(6)}$. 
En relación al autotrasplante, la mayoría de los estudios utilizan caninos o premolares, siendo la mayor de sus complicaciones, la reabsorción radicular y anquilosis del diente trasplantado ${ }^{(7)}$.

Por último, la utilización de un puente adhesivo de un ala, cementado con resina podría ser una alternativa si el espacio disponible es reducido. Esta opción requiere de una preparación mínima, pero hay que considerar la elección del material, la superficie de unión, la técnica, el espesor del conector y su mantención ${ }^{(3)}$.

En pacientes jóvenes hay que tener precaución a la hora de optar por un tratamiento, ya que cualquier rehabilitación definitiva, puede traer consecuencias a futuro(8). Debido a esto último, es que se realizó esta revisión bibliográfica, que tuvo como objetivo, describir y comparar los diferentes enfoques terapéuticos que existen actualmente para resolver esta problemática, junto con sus resultados a largo plazo.

\section{METODOLOGÍA}

Se realizó una búsqueda electrónica de la evidencia disponible, en las bases de datos Pubmed, EBSCO Dentistry \& Oral Sciences Source y The Wiley-Blackwell Online Library. Se utilizaron palabras clave tales como: "Lateral Incisors", "Agenesis", "Treatment" relacionadas entre sí con el término booleano "AND".

Para la búsqueda específica en Pubmed, se agregaron los términos $\mathrm{MeSH}$ "Incisors[MeSh]" , "Anodontia"[Mesh] y "Therapy [MeSH]" y se combinaron de la siguiente manera: ((Incisor[MeSH Terms]) AND (Anodontia[MeSH Terms])) AND (Therapy[MeSH Terms]).

Se filtraron los resultados por fecha, desde enero del 2000, hasta junio del 2020. Se leyeron los títulos y resúmenes, y se incluyeron los artículos correspondientes a revisiones sistemáticas, estudios de caso y control, estudios retrospectivos observacionales, ensayos clínicos, revisiones narrativas y estudios de cohorte, reportes de casos y estudios comparativos. Estudios que consideraran tanto pacientes adolescentes como adultos, sin límite de edad y artículos escritos en inglés y español. Se consideraron los artículos que describen el tratamiento de incisivo lateral superior. La unidad de estudio corresponde a humanos y animales. Fueron excluidos los estudios correspondientes a resúmenes de congresos, artículos de opinión y guías clínicas. Además, no fueron considerados los artículos que describieron tratamientos de agenesia en otros dientes que no fuesen incisivos laterales superiores.

Por último, para la correcta alineación de las piezas en las arcadas, la técnica utilizada fue de arco recto con aparatos fijos multibrackets.

\section{RESULTADOS}

La búsqueda realizada en Pubmed arrojó 49 resultados, luego se filtró por título obteniendo 20 artículos, para luego utilizar un tercer filtro determinado por el abstract, quedando 2 artículos seleccionados. EBSCO Dentistry and Oral Sciences Source arrojó 147 resultados, de los cuales en el primer filtro se seleccionaron 57 artículos, y con el segundo filtro 18 artículos finales. En Wiley-Blackwell Online Library se obtuvieron 381 resultados, de los cuales se seleccionaron con el primer filtro 18 artículos, y finalmente con el segundo filtro con 11 artículos finales, donde solamente se utilizaron 6 por repetición en las otras bases de datos.

Sumado a los resultados que se obtienen en la búsqueda a partir de los filtros mencionados, se agregan 11 artículos a partir de una búsqueda manual retrógrada.

Quedando un total de 37 artículos finales, de los que 5 corresponden a revisiones sistemáticas, 7 reportes de caso, 6 estudios retrospectivos observacionales, 2 estudios prospectivos observacionales, 1 ensayo clínico, 11 revisiones narrativas, 4 estudios de cohorte y 1 estudio comparativo. (Tabla 1)

1. Cierre de espacio mediante aparatología fija de ortodoncia para reemplazo del incisivo lateral maxilar faltante por el canino permanente.

El cierre de espacios mediante aparatología fija consiste en el movimiento mesial del canino permanente para lograr ubicarlo en la posición del incisivo lateral faltante. Esta técnica requiere de una comunicación efectiva con el odontólogo rehabilitador para alcanzar la estética óptima de la sonrisa. El canino maxilar suele tener un color más amarillento que el incisivo central, de modo que lo más probable es que necesite de blanqueamiento vital para asemejarse a sus dientes adyacentes $s^{(9,10,11,12,13,1)}$. Debido a su anatomía, la modificación de su forma será necesaria y requerirá preparaciones idealmente mínimamente invasivas de carillas cerámicas o de resina compuesta ${ }^{(13)}$. (Figura 1)

2. Apertura de espacio y posterior instalación de implantes oseointegrados.

Actualmente, el uso de implantes oseointegrados para reemplazar
Tabla 1.
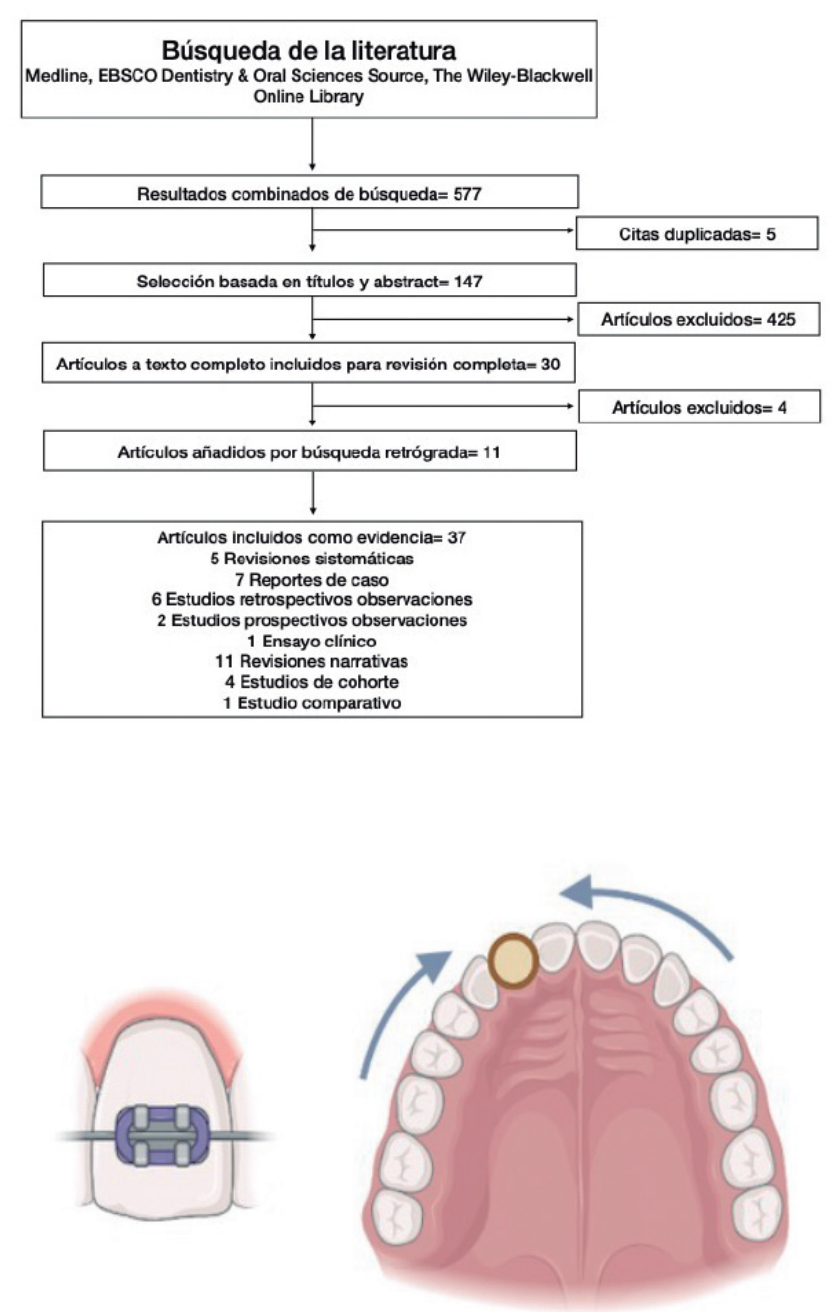

Figura 1.

piezas dentarias ausentes es muy utilizado como tratamiento rehabilitador, gracias a su alta tasa de supervivencia descrita en la literatura. El dispositivo protésico actúa como un diente anquilosado, por lo que no continúa con el crecimiento y velocidad de desarrollo de los procesos dentoalveolares de forma natural| ${ }^{(9,14,5,13,15)}$. El crecimiento en el maxilar, se produce en sentido anteroinferior, a partir de aposición ósea en las tuberosidades y suturas adyacentes ${ }^{(16)}$. El tratamiento con implantes debe ser desarrollado una vez que el crecimiento craneofacial haya terminado. Se ha observado que el final de la adolescencia y el comienzo de la edad adulta coinciden con una disminución en el peak de crecimiento. La cantidad de crecimiento disminuye después de los 20 años, sin embargo, continúa en el esqueleto craneofacial. Los estudios de Bjork determinaron que el maxilar crece aproximadamente $3 \mathrm{~mm}$ en un periodo de 2 años en el peak puberal|(17). La infraoclusión es la principal complicación relacionada con la instalación de implantes en el sector anterior y se ha demostrado que ocurre independiente de la edad, es decir, continúa incluso después de la segunda y tercera década, pero con un rango menor a $0,5 \mathrm{~mm}^{(18)}$.

Una opción novedosa para la preservación de los procesos alveolares, corresponde a la utilización de dispositivos de anclaje temporal o microtornillos ${ }^{(19)}$

Se ha observado que instalando un microtornillo transcortical se preservó el área vestíbulo-lingual total del proceso alveolar, por lo tanto, esto parece indicar que su uso mantiene el ancho de los procesos alveolares, previniendo o reduciendo la atrofia ósea en el área edéntula. Además, mantiene tanto la calidad como la cantidad del hueso, reduciendo la necesidad de cirugía de reconstrucción antes de la inserción de los implantes. Si bien los microtornillos microscópicamente no están oseointegrados, tampoco siguen el desarrollo de los procesos alveolares durante la erupción de los dientes adyacentes, por lo tanto, aún no hay casos en que se demuestre el éxito en relación al desarrollo vertical de los procesos alveolares ${ }^{(19-21)}$. (Figura 2) 


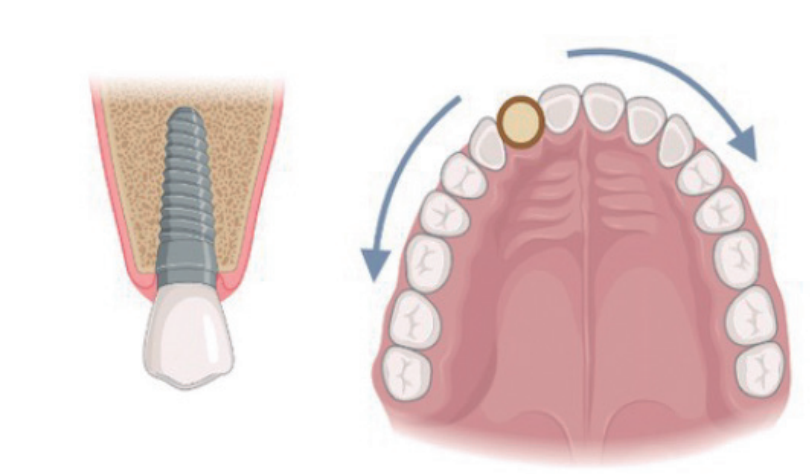

Figura 2.

\section{Autotrasplante dental.}

El autotrasplante consiste en la utilización de un diente natural del mismo paciente, que es colocado en un sitio previamente preparado en donde existe ausencia de una pieza dentaria. En esta terapia, uno de los factores más importantes para el éxito corresponde al grado de desarrollo radicular del diente a trasplantar, siendo los de mejor pronóstico aquellos que están en estadio 3 o 4 de Nolla ${ }^{(7)}$; o con formación de los $2 / 303 / 4$ de la longitud total de la raíz, con un ápice abierto mayor a $1 \mathrm{~mm}$, con el fin de conseguir una correcta revascularización apical ${ }^{(22)}$. En cuanto al diente a seleccionar, se suele considerar a los premolares como el injerto más prometedor, debido a su morfología radicular. En cuanto a manejo, la cirugía debe ser cuidadosa, sin dañar la superficie radicular(7), conservando la vaina epitelial de Hertwig del diente en desarrollo.

Un factor crítico para el éxito depende del uso de una técnica aséptica y una cirugía atraumática(23), junto con una buena estabilidad primaria, siendo necesario ferulizar el diente trasplantado. La sutura fue el material mejor reportado para este fin, ya que permite el micromovimiento natural del diente evitando una posible anquilosis.

El beneficio del autotrasplante, consiste en que se utiliza un suplemento biológico que es capaz de regenerar el ligamento periodontal y el hueso alveolar de forma normal, con la consiguiente formación radicular total. Aún si el diente trasplantado falla, ayuda a que se mantenga la oclusión y continúe la formación del hueso alveolar ${ }^{(9,7)}$. El diente trasplantado seguirá el crecimiento y la velocidad normal del hueso alveolar en conjunto con sus piezas adyacentes ${ }^{(23)}$. Se debe considerar a la vez, que el diente deberá recibir rehabilitación posterior. (Figura 3)

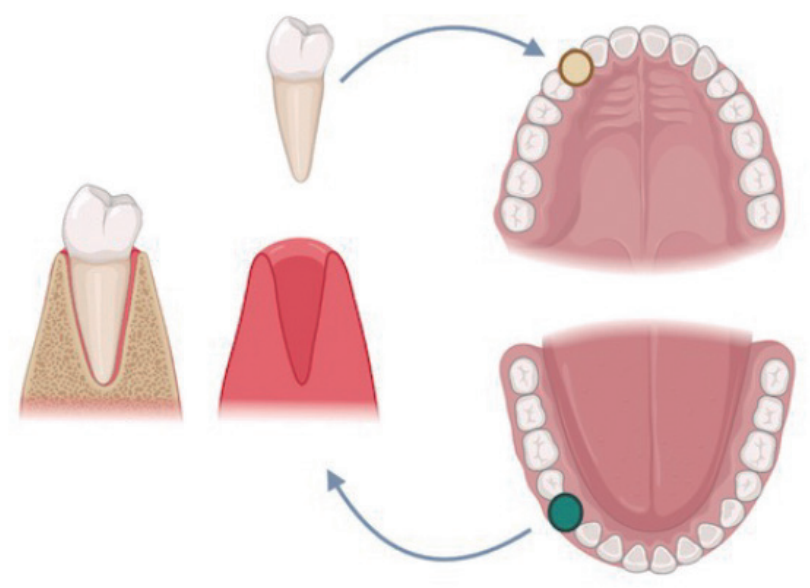

Figura 3.

\section{Rehabilitación con pónticos.}

La rehabilitación protésica de la agenesia de incisivos laterales se puede lograr mediante aparatos fijos o removibles. La virtud de los aparatos fijos por sobre los removibles es la estética, debido a que los segundos presentan retenedores metálicos en piezas adyacentes $\mathrm{y}$, además, el paciente debe quitárselos y permitir el descanso del periodonto. Dentro de los aparatos fijos tenemos prótesis fija plural, que abarca los dos dientes adyacentes, con desgastes excesivos y poco conservadores, y prótesis adhesivas de una y dos alas unidas con resina, que a partir de preparaciones mínimamente invasivas de la pieza adyacente logran una buena adhesión al sustrato dentario, sin necesidad de desgastar gran cantidad de tejido sano(24,25). Los biomateriales para poder realizar esta rehabilitación pueden ser estructuras metálicas revestidas de cerámica o resina y no revestidas, de metal (zirconio altamente sinterizado revestido o cerámica reforzada con vidrio), o estructuras all composite ${ }^{(25)}$.

En cuanto al diseño, existen dos tipos, con una o dos alas. Se observa, en general, que aquellos de un ala de cerámica sin metal, muestran una excelente longevidad, teniendo mejor éxito clínico en comparación a los diseñados con dos retenedores palatinos de disilicato de litio. Por lo tanto, la tasa de sobrevivencia de aquellos de un ala, puede ser superior a la de dos alas ${ }^{(26)}$. (Figura 4)
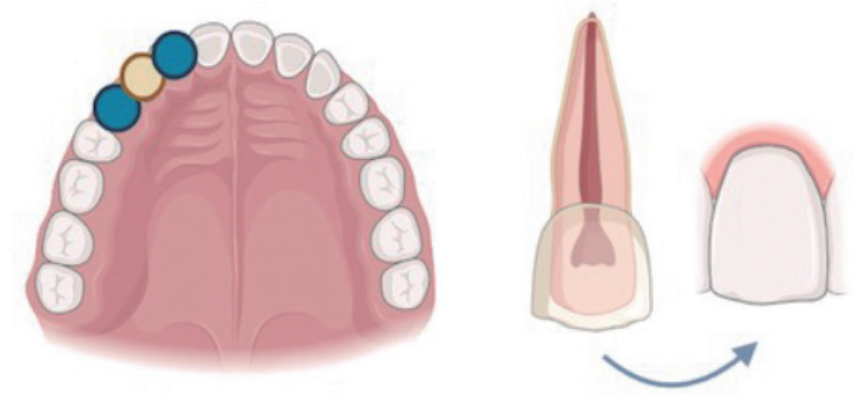

Figura 4.

\section{DISCUSIÓN}

El maxilar y la mandíbula comienzan su desarrollo alrededor de la séptima semana intrauterina. El estímulo para la formación de los procesos alveolares está dado por los dientes en crecimiento, es decir, la pared de los alvéolos comienza a desarrollarse al completarse la formación coronaria del diente y se inicia el crecimiento de la raíz del folículo dentario. De esta forma, es importante entender que los procesos alveolares son una estructura "odontodependiente", es decir, se atrofian si el diente es extraído o no se desarrolla en el caso de alguna anomalía ${ }^{(27)}$.

Stumbras et al., mencionó que ciertos estudios demostraron que la pérdida de hueso alveolar durante los primeros 12 meses después de la extracción del diente fue de $11-22 \%$ de altura y $29-63 \%$ de ancho, mientras que $2 / 3$ de la cresta ósea se pierde durante los primeros 3 meses luego de la extracción. Por lo general, la pérdida ósea es mayor en relación a la tabla vestibular, y en pacientes con biotipo fino ${ }^{(28)}$.

Una opción para la preservación es la utilización de mini-tornillos de ortodoncia, tales como los implantes mini-screw (MSI), mini-placa y palatinos. Su uso es para preservar de forma adecuada el reborde alveolar, hasta que el paciente tenga la edad suficiente para recibir un implante oseointegrado(4).

En cuanto a la instalación de implantes oseointegrados Kavadia et al, Kiliaridis et al,Richardson et al, Dorigatti et al, y Antonarakis et al, coincidieron en que su uso era la alternativa más conservadora, ya que los dientes vecinos no necesitaban cambios y quedaban sin secuelas a causa de la rehabilitación, lo que cobraba especial importancia en pacientes jóvenes ${ }^{(9,10,21,29,5)}$.

Dorigatti et al, destacó que es necesario hacer este tipo de tratamientos, una vez que el crecimiento cráneo-facial haya terminado(29), postura coincidente con la de Priest et al, quien añadió que en los sitios donde iba el implante, los procesos alveolares debían estar desarrollados completamente ${ }^{(8)}$. Dorigatti et al, mencionaba, además, que había que tener especial cuidado con el tiempo planeado para la apertura del espacio, con el fin de que estuviera disponible la mayor cantidad de hueso y se generara un adecuado espacio mesiodistal entre coronas y raíces de los dientes adyacentes ${ }^{(29)}$. Sumado a esto, Kavadia et al, destacó las ventajas del uso de implantes, dentro de las cuales estos permitían la mantención del hueso alveolar, mejoraban la función oclusal, brindaban una estética aceptable, en conjunto con un tratamiento de ortodoncia que solía ser durante un corto periodo de tiempo y relativamente simple ${ }^{(9)}$. Respecto a la infraoclusión, la mayoría de los autores coincidieron que era la principal desventaja. Plakwicz et al, lo asociaba a la continua erupción de los dientes vecinos, independiente de la edad del paciente, lo que además traía consigo la reabsorción de la cortical vestibular, junto con pérdida del hueso marginal de las piezas vecinas $^{(20)}$. Nilsson et al, analizó la prevalencia de infraposición de los implantes en el sector anterior, tanto en adolescentes como en adultos, obteniendo como resultado que esta ocurría independiente de la edad a la que se colocaba el implante, y que solía suceder en la mayoría de 
los pacientes, aunque en un rango menor a $0,5 \mathrm{~mm}^{(18)}$. Andersson et al, concluyó que había una mayor predisposición en el género femenino ${ }^{(30)}$ postura similar a la de Schneider et al, que lo asoció al fenotipo facial divergente, común en el género femenino(1). Cope et al, planteó el uso de microtornillos con la finalidad de preservar el hueso alveolar, evitando posteriores injertos de huesos y/o tejidos blandos. Estos se usaban como una solución temporal, durante la etapa de crecimiento del paciente y luego eran reemplazados por el implante. Sin embargo, a la fecha no hay ensayos clínicos que respaldaran esta postura(4).

Zarone et al, llevó a cabo un seguimiento de 24 a 39 meses, determinando que durante un año postcarga la pérdida ósea periimplantaria era de un $0,6 \mathrm{~mm}$ en promedio y luego de 12 meses 0,2 $\mathrm{mm}$ por año(31). Thilander et al, planteó que la reabsorción ósea era de $0,1 \mathrm{~mm}$ al año de carga y los valores continuaban similares los años siguientes ${ }^{(13)}$. Branzen et al, expuso, que el promedio de reabsorción era de $0,1 \mathrm{~mm}$ al año, coincidiendo con los autores anteriormente mencionados $^{(32)}$. En cuanto a problemas periodontales, Silveira et al, describió que existía una retracción de la encía vestibular y un relleno incompleto de la papila interdental asociado al implante ${ }^{(33)}$. De forma similar, Thilander et al, describió casos en donde era posible apreciar una decoloración de la mucosa, con tonalidad azul, lo que indicaría una pérdida de hueso marginal vestibular, afectando estéticamente a los pacientes y por sobre todo, a aquellos que tenían una línea de la sonrisa alta(13). Kavadia et al, añadió que podía ocurrir una exposición del metal o porcelana subyacente a la corona del implante(9). Contrariamente, Dierens et al, evalúo la posición de los tejidos blandos en relación a los implantes, observando que estos se mantenían estables en el tiempo(34).

En cuanto a la sobrevida de los implantes, Branzén et al, realizó un seguimiento de 4 a 9 años en 36 pacientes, donde se instalaron implantes a temprana edad. La sobrevida fue de un $100 \%$, sin embargo, se consideró que este estudio tiene una muestra muy poco representativa ${ }^{(32)}$. Otra consideración es descrita por Antonarakis et al, asociando los implantes con infraoclusión, incluso en pacientes adultos, trayendo grandes problemas estéticos, determinando que el costo-efectividad aumentaba debido a la necesidad de realizar cirugías posteriores para el remodelado de los tejidos ${ }^{(5)}$.

Respecto a la postura del paciente, esta técnica parecía ser de elección únicamente en pacientes adultos, logrando grandes niveles de estética y funcionalidad, pese a que el tratamiento incluía una planificación y ejecución más lenta y de mayor complejidad( ${ }^{(8)}$.

Por otro lado, para el cierre de espacio con ortodoncia y sustitución canina, los autores concordaban que existían parámetros que se debían evaluar entre las cuales se incluyó el perfil facial, el tipo de maloclusión, presencia de apiñamiento dentario, línea de la sonrisa, dimensiones caninas y color. Las indicaciones para cierre de espacio de ortodoncia eran claras: pacientes que presentaban apiñamiento severo mandibular, y anterior con relación molar de clase I y también una relación molar de clase II bilateral, sin apiñamiento y protrusión dental en el segmento anterior mandibular ${ }^{(10)}$. Kiliaridis et al, mencionaba que la principal ventaja del cierre de espacios correspondía a la longevidad y predictibilidad del resultado terapéutico con la posibilidad de finalizar el tratamiento dentro de los primeros años de la adolescencia. En cuanto a costos, el cierre de espacios era más económico. Como desventaja, expuso que la reducción coronaria canina que se necesitaba posterior a la ortodoncia, para simular la arquitectura del incisivo lateral requería de gran desgaste de estructura dental, sumado a la necesidad de aumentar de tamaño los incisivos centrales en algunos casos. Se debía considerar también el blanqueamiento dentario del canino por la diferencia de color con los incisivos centrales, o un tratamiento restaurador de la corona. En cuanto a los tejidos blandos, era necesario ubicar de manera adecuada el zenit gingival ${ }^{(10)}$. Silveira et al, obtuvo como resultados, que ninguna rehabilitación con porcelana o puentes unidos con resina, tenía mejores índices periodontales que el cierre de espacio con ortodoncia.

Rafalowicz et al, mencionó que el grupo que se sometió a cambio de forma del canino posterior a cierre de espacios con ortodoncia, en 4 años presentó casos de decoloración inaceptables y alteraciones de adhesión del material. Explicó, además, que cambiar la forma canina tenía un efecto aceptable en más de dos tercios de casos revisados ${ }^{(35)}$.

Actualmente, con el uso de dispositivos de anclaje temporal (TAD), ha logrado dar resultados favorables a casos ortodónticos complejos, como expuso Amm et al, quien reportaba que cerrar los espacios a causa de agenesia en incisivos laterales, en conjunto con el uso de TAD, proporcionaban resultados satisfactorios, gracias a que mantenían el reborde alveolar adecuado, incluso cuando el paciente tenía perfil cóncavo(36)

Kavadia et al, describió que la gran ventaja de esta terapia correspondía a la permanencia de los resultados de tratamiento a través del tiempo. Además, la arquitectura gingival y del hueso alveolar podían establecerse gracias al movimiento mesial del canino, siendo la dentición natural la que mantenía o preservaba de mejor manera la cantidad de hueso alveolar en la región. En cuanto a las desventajas, la sustitución canina tenía tendencia a reabrir los espacios, por lo que se requería del uso de retenedores ${ }^{(9)}$. Wagner et al, describió el tratamiento de 37 pacientes, que posteriormente se trató con remodelado de las piezas dentarias con resina compuesta. Los resultados clínicos fueron aceptables en un $100 \%$ de los pacientes en los años $1-3,93 \%$ de los pacientes en los años $4-7$, un $78,5 \%$ de los pacientes en el año $8, y$ $73,2 \%$ en el año 9 y $62,5 \%$ en el año 10 . Sin embargo, hay que tener en consideración que la remodelación del canino por método directo puede requerir de mantenimiento de rutina o nueva aplicación de resina compuesta $^{(12)}$. Schneider et al, mencionó que el cierre de espacio es un tratamiento óptimo a largo plazo cuando se lograba la extrusión diferencial de los caninos e intrusión de premolares, sumado al blanqueamiento y reconstrucción de los seis dientes anteriores y la curvatura bucal. Cabe destacar que esta técnica es señalada por el autor como la de elección, tanto por clínicos como por los mismos pacientes, a la hora de tratarse de niños en crecimiento o adultos jóvenes, gracias a que permite además de una buena estética, una gran estabilidad oclusal y funcional(2). Similar es la postura de Priest et al., coincidiendo en que es la técnica apropiada a la hora de buscar rapidez y efectividad ${ }^{(24)}$.

En cuanto al autotrasplante dental, Antonarakis et al, expuso que si bien, en su análisis de costo-efectividad era el mejor evaluado, se trataba de una terapia que no era aplicable a todos los casos. Debía ser considerado, cuando existía la indicación de extracción de premolares, o cuando la extracción de un premolar resolvía algún problema funcional y estético en la zona anterior, creando solo un problema menor en el sitio donante, pudiendo corregirlo con ortodoncia. Además, era de suma importancia el tiempo en este procedimiento, ya que requería del desarrollo radicular en $2 / 3 \circ 3 / 4$ de su total. Las ventajas que presentaba eran que se debía a un reemplazo biológico, con una posición ajustable y que se insertaba en el hueso con su ligamento periodontal normal(5). Stenvik et al, menciona que el plan de tratamiento debía considerar la necesidad de ortodoncia pre y post trasplante. En cuanto al diente a seleccionar, los premolares solían ser el donante preferido, ya que la morfología de la raíz era recta y cónica, sumando que el espacio de extracción podía utilizarse para aliviar el apiñamiento. La morfología del primer premolar inferior, era la que ofrecía la mejor oportunidad. Posteriormente se debía contornear el diente para simular el incisivo maxilar y lograr una estética adecuada. El resultado del análisis sistemático después del autotrasplante de premolares demostró una tasa de supervivencia a largo plazo de más del $90 \%$. En cuanto a la estética también los resultados fueron satisfactorios, y en el caso de las complicaciones, se asociaban a la necesidad de realizar restauraciones de composite para remodelar la pieza dentaria(37).

Sobre la rehabilitación con pónticos adhesivos, Kiliaridis et al, mencionó que esta constituía la opción más conservadora, porque los dientes adyacentes se sometían a una preparación dental mínima(10). Así mismo, Priest et al, expusó que los puentes de resina favorecían la mantención de la papila dentaria y evitaban que fuerzas excesivas afectaran la cresta ósea. Mencionó, además, que podía ser removido con una alteración mínima de la estructura dentaria adyacente(24). En cuanto a las ventajas, Kiliaridis et al, describió que evitaban el trauma pulpar, no necesitaba preparaciones subgingivales, era menos costoso y tomaba menos tiempo clínico. Sin embargo, el clínico debía considerar la posición vertical de los dientes, la inclinación de los incisivos y que no existiera movilidad dentaria ${ }^{(10)}$. Como desventaja, Silveira et al, mencionó que los puentes tienen peores condiciones periodontales, debido a la posibilidad de contornos excesivos y malposiciones de los dientes en relación a estas rehabilitaciones, dificultando la higiene ${ }^{(33)}$. En cuanto a sobrevida, Garnett et al, realizó un seguimiento de 11 años en 45 pacientes, donde un $58,9 \%$ de los puentes se mantuvieron intactos en boca, generalmente en pacientes adultos, asociándolo a que, en pacientes jóvenes, es más común que ocurrieran fallas, debido a que tenían coronas clínicas más cortas y, por ende, una menor área de cementación ${ }^{(25)}$. Antonarakis et al, en su estudio de costo-efectividad encontró que, a menudo, al corto plazo, el costo era menor comparado con las otras alternativas terapéuticas, sin embargo, a largo plazo era cuestionable, debido a que este tipo de rehabilitación tenía una menor durabilidad ${ }^{(5)}$.

\section{CONCLUSIÓN}

Se puede determinar entonces que no existe una alternativa terapéutica mejor que otra para el tratamiento de la agenesia del incisivo lateral superior. La elección adecuada depende del paciente, su contexto y caso clínico particular, de manera de poder entregarle el resultado más eficiente y duradero en el tiempo. Se requiere la realización de ensayos clínicos aleatorizados que le otorguen mayor grado de evidencia a la 
literatura y así definir una pauta con mejor claridad.

\section{LIMITACIONES}

Los resultados obtenidos de esta revisión deben ser analizados con cautela, debido al tipo de literatura disponible actualmente sobre el tema expuesto que en su mayoría corresponde a revisiones narrativas y estudios de casos. Además, la elección de una alternativa de tratamiento depende de las variables individuales de cada caso en particular, por lo cual no existe la posibilidad de establecer una guía para el clínico que le permita optar por un tipo de tratamiento.

\section{RELEVANCIA CLÍNICA}

La agenesia de incisivos laterales superiores corresponde a una de las más frecuentes y trae consigo un compromiso estético y funcional que requiere manejo multidisciplinario. Para lograrlo, se debe tener un completo conocimiento de las opciones terapéuticas disponibles, lo cual justifica científicamente la realización de esta revisión. Dentro de los resultados principales, existen diferentes alternativas terapéuticas para la sustitución de estos dientes, como por ejemplo, el cierre de espacios mediante ortodoncia, rehabilitación con implante, autotrasplante y pónticos adhesivos. Como consecuencia práctica el clínico podrá contar con herramientas terapéuticas que le permitan rehabilitar la ausencia congénita de estas piezas dentarias.

\section{CONFLICTO DE INTERÉS}

El autor declara no tener potencial conflicto de interés respecto a la autoría y publicación de este artículo.

\section{ANEXO 1}

Tabla 1: Publicaciones incluidas, número de participantes, tratamiento evaluado, resultados y tipo de estudio.

\begin{tabular}{|c|c|c|c|c|}
\hline Publicación. & $\begin{array}{l}N^{\circ} \text { de partici- } \\
\text { pantes. }\end{array}$ & Tratamiento evaluado. & Outcome. & Tipo de estudio. \\
\hline $\begin{array}{l}\text { Schneider et al. } \\
(2020)\end{array}$ & 2 pacientes. & $\begin{array}{l}\text { Apertura de espacio y posterior } \\
\text { instalación de implante oseoin- } \\
\text { tegrado diente } 1.2 \text {. } \\
\text { Apertura de espacio y posterior } \\
\text { instalación de implante oseoin- } \\
\text { tegrado diente } 2.5 \text {. }\end{array}$ & $\begin{array}{l}\text { Excelente estética, salud peri-implantar, recesión } \\
\text { gingival. } \\
\text { Apertura de espacio con buen control vertical, e inclina- } \\
\text { ción de los incisivos dentro de los límites normales. } \\
\text { Disminución del riesgo de infraoclusión, dehiscencias } \\
\text { óseas, recesión gingival, periimplantitis. }\end{array}$ & Reporte de caso. \\
\hline $\begin{array}{l}\text { Schneider et al. } \\
\text { (2018) }\end{array}$ & 32 pacientes & $\begin{array}{l}\text { Cierre de espacio mediante } \\
\text { aparatología fija de ortodoncia. } \\
\text { Apertura de espacio y posterior } \\
\text { instalación de implante oseoin- } \\
\text { tegrado. }\end{array}$ & $\begin{array}{l}\text { Notables diferencias de color que requirieron blanquea- } \\
\text { mientos a repetición. } \\
2 \text { pacientes con caninos desvitalizados luego del movi- } \\
\text { miento mesial. } \\
5 \text { pacientes requirieron reparación de restauraciones de } \\
\text { resina compuesta. } \\
4 \text { pacientes necesitaron reparación de cúspides en } \\
\text { premolares. } \\
22 \text { recesiones gingivales (14 en PM). } \\
\text { PES total medio } 9,39 \text { y WES } 8,36 \text {. } \\
\text { Implantes integrados sin supuración en el surco o } \\
\text { signos de inflamación de los tejidos. } \\
\text { No se produjeron fallos protésicos. } \\
\text { Profundidades de saco entre } 2-3 \text { mm, con un promedio } \\
\text { de } 2,24 \text { mm. } \\
9 \text { recesiones gingivales. } \\
\text { PES total medio de } 8,91 \text { y un WES } 8,72 \text {. } \\
\text { Los pacientes de ambos grupos estaban satisfechos } \\
\text { con los resultados. }\end{array}$ & $\begin{array}{l}\text { Estudio retros- } \\
\text { pectivo observa- } \\
\text { cional. }\end{array}$ \\
\hline $\begin{array}{l}\text { Cocconi et al. } \\
(2020)\end{array}$ & - & $\begin{array}{l}\text { Cierre de espacio mediante } \\
\text { aparatología fija de ortodoncia, } \\
\text { versus, apertura de espacio y } \\
\text { posterior instalación de implan- } \\
\text { te oseointegrado. }\end{array}$ & $\begin{array}{l}\text { Se recomienda el cierre de espacios en agenesias } \\
\text { unilaterales, cuando el incisivo contralateral sea } \\
\text { extraído, siempre y cuando se modifiquen los dientes } \\
\text { anteriores, para cumplir con los parámetros estéticos, o } \\
\text { cuando exista una maloclusión en el mismo lado de la } \\
\text { agenesia. } \\
\text { En pacientes que se realiza apertura de espacio, se re- } \\
\text { comienda usar materiales provisorios, durante el tiempo } \\
\text { necesario, hasta que el paciente llegue a la adultez. }\end{array}$ & $\begin{array}{l}\text { Revisión narra- } \\
\text { tiva. }\end{array}$ \\
\hline $\begin{array}{l}\text { Cope et al. } \\
(2014)\end{array}$ & 2 pacientes. & $\begin{array}{l}\text { Apertura de espacio y pos- } \\
\text { terior instalación de implante } \\
\text { oseointegrado: Microtornillos } \\
\text { de ortodoncia. }\end{array}$ & $\begin{array}{l}\text { El uso de implantes de microtornillos de ortodoncia trae } \\
\text { beneficios en el paciente tanto para el hueso alveolar } \\
\text { como para los tejidos blandos, a diferencia de otros } \\
\text { tratamientos que pueden causar atrofia ósea, lo que } \\
\text { trae problemas para la posterior rehabilitación con } \\
\text { implantes. }\end{array}$ & Reporte de caso. \\
\hline
\end{tabular}


Tabla 1: Publicaciones incluidas, número de participantes, tratamiento evaluado, resultados y tipo de estudio. (continuación)

\begin{tabular}{|c|c|c|c|c|}
\hline $\begin{array}{l}\text { Antonarakis et } \\
\text { al. (2014) }\end{array}$ & - & $\begin{array}{l}\text { Apertura de espacio y pos- } \\
\text { terior instalación de implante } \\
\text { oseointegrado; Rehabilitación } \\
\text { con pónticos; Autotrasplante } \\
\text { dental. }\end{array}$ & $\begin{array}{l}\text { La alternativa con una mayor relación costo/efectividad } \\
\text { era el autotrasplante dental, mientras que la con menor } \\
\text { valoración corresponde al puente de cobertura comple- } \\
\text { ta. Sin embargo, la elección terapéutica dependerá de } \\
\text { factores como la edad del paciente, tipo de dentición, } \\
\text { oclusión y conservación dentaria. }\end{array}$ & $\begin{array}{l}\text { Revisión narra- } \\
\text { tiva. }\end{array}$ \\
\hline $\begin{array}{l}\text { Rosa et al. } \\
(2020)\end{array}$ & 2 pacientes. & $\begin{array}{l}\text { Cierre de espacio mediante } \\
\text { aparatología fija de ortodoncia. }\end{array}$ & $\begin{array}{l}\text { El cierre de espacios tiene como ventajas un menor } \\
\text { tiempo de tratamiento, muy buena evidencia científica, } \\
\text { la salud periodontal es más predecible que en casos } \\
\text { donde se sustituye el diente ausente, es un tratamiento } \\
\text { factible en maloclusiones. Es importante un tratamiento } \\
\text { multidisciplinario, con el fin de lograr una buena estéti- } \\
\text { ca, función y estabilidad. }\end{array}$ & Reporte de caso. \\
\hline $\begin{array}{l}\text { Vilhjálmsson et } \\
\text { al. (2011) }\end{array}$ & 31 pacientes. & Autotrasplante dental. & $\begin{array}{l}\text { El autotrasplante es considerado una alternativa de alto } \\
\text { éxito en pacientes jóvenes en crecimiento. Se reco- } \\
\text { mienda usar dientes que tengan su raíz en desarrollo, } \\
\text { por sobre aquellos que ya completaron la formación } \\
\text { de ella. Se recomienda hacer un seguimiento de estos } \\
\text { dientes de al menos } 5 \text { años. }\end{array}$ & $\begin{array}{l}\text { Estudio de } \\
\text { cohorte. }\end{array}$ \\
\hline $\begin{array}{l}\text { Priest et al. } \\
(2019)\end{array}$ & - & $\begin{array}{l}\text { Apertura de espacio y posterior } \\
\text { instalación de implante oseoin- } \\
\text { tegrado. }\end{array}$ & $\begin{array}{l}\text { La sustitución de dientes ausentes por implantes es } \\
\text { una buena alternativa, en términos de función y estéti- } \\
\text { ca, pero necesita una planificación y una ejecución más } \\
\text { compleja que el resto de las alternativas. }\end{array}$ & $\begin{array}{l}\text { Revisión narra- } \\
\text { tiva. }\end{array}$ \\
\hline $\begin{array}{l}\text { Kavadia et al. } \\
(2011)\end{array}$ & - & $\begin{array}{l}\text { Cierre de espacio mediante } \\
\text { aparatología fija de ortodoncia; } \\
\text { Apertura de espacio y posterior } \\
\text { instalación de implante oseoin- } \\
\text { tegrado. }\end{array}$ & $\begin{array}{l}\text { Es necesario un manejo multidisciplinario, cada técnica } \\
\text { tiene sus diferentes ventajas y desventajas, indicacio- } \\
\text { nes y limitaciones. La elección depende de la base del } \\
\text { clínico y las expectativas del paciente. }\end{array}$ & $\begin{array}{l}\text { Revisión narra- } \\
\text { tiva. }\end{array}$ \\
\hline $\begin{array}{l}\text { Kiliaridis et al. } \\
(2016)\end{array}$ & - & $\begin{array}{l}\text { Cierre de espacio mediante } \\
\text { aparatología fija de ortodoncia; } \\
\text { Alternativas rehabilitadoras. }\end{array}$ & $\begin{array}{l}\text { Ambas alternativas se consideran efectivas, sin embar- } \\
\text { go, el cierre de espacios con ortodoncia pareciera ser } \\
\text { más ventajoso sobre las alternativas rehabilitadoras. Se } \\
\text { necesitan más estudios retrospectivos y prospectivos, } \\
\text { para una mejor conclusión. }\end{array}$ & $\begin{array}{l}\text { Revisión siste- } \\
\text { mática. }\end{array}$ \\
\hline $\begin{array}{l}\text { Park et al. } \\
(2010)\end{array}$ & 1 paciente. & $\begin{array}{l}\text { Cierre de espacio mediante } \\
\text { aparatología fija de ortodoncia. }\end{array}$ & $\begin{array}{l}\text { Tanto la alternativa de cierre de espacio, como la } \\
\text { apertura de este y posterior rehabilitación con implantes } \\
\text { oseointegrados son válidas, si hay comunicación entre } \\
\text { ortodoncistas y rehabilitadores. }\end{array}$ & Reporte de caso. \\
\hline $\begin{array}{l}\text { Wagner et al. } \\
(2019)\end{array}$ & 37 pacientes. & $\begin{array}{l}\text { Cierre de espacio mediante } \\
\text { aparatología fija de ortodoncia, } \\
\text { y remodelado canino. }\end{array}$ & $\begin{array}{l}\text { El estudio clínico muestra que el cierre de espacio } \\
\text { otorga una oclusión en función de grupo aceptable, que } \\
\text { no afecta la ATM y preserva la salud periodontal. } \\
\text { Las restauraciones de los caninos realizadas con méto- } \\
\text { do directo requieren mantención y re-aplicación. }\end{array}$ & $\begin{array}{l}\text { Estudio prospec- } \\
\text { tivo observacio- } \\
\text { nal. }\end{array}$ \\
\hline $\begin{array}{l}\text { Thilander et al. } \\
\text { (2008) }\end{array}$ & - & $\begin{array}{l}\text { Cierre de espacio mediante } \\
\text { aparatología fija de ortodoncia, } \\
\text { versus, apertura de espacio y } \\
\text { posterior instalación de implan- } \\
\text { te oseointegrado. }\end{array}$ & $\begin{array}{l}\text { Ambas alternativas presentan ventajas y desventajas, } \\
\text { por lo que hacen falta más estudios. }\end{array}$ & $\begin{array}{l}\text { Revisión siste- } \\
\text { mática. }\end{array}$ \\
\hline $\begin{array}{l}\text { Bernard et al. } \\
(2004)\end{array}$ & 28 pacientes. & $\begin{array}{l}\text { Apertura de espacio y posterior } \\
\text { instalación de implante oseoin- } \\
\text { tegrado. }\end{array}$ & $\begin{array}{l}\text { Puede haber un crecimiento vertical en pacientes } \\
\text { adultos que hayan recibido implantes oseointegrados } \\
\text { en el sector anterior. Sin embargo, son pocos los casos } \\
\text { donde persiste un crecimiento residual y las alteracio- } \\
\text { nes suelen ser mínimas. }\end{array}$ & $\begin{array}{l}\text { Estudio retros- } \\
\text { pectivo. observa- } \\
\text { cional. }\end{array}$ \\
\hline $\begin{array}{l}\text { Coccheto et al. } \\
(2019)\end{array}$ & 94 pacientes. & $\begin{array}{l}\text { Apertura de espacio y posterior } \\
\text { instalación de implante oseoin- } \\
\text { tegrado. }\end{array}$ & $\begin{array}{l}\text { La infraoclusión se presentó en un } 73 \% \text { de los casos, } \\
\text { sin mayores diferencias entre sexo y edad. Por lo que } \\
\text { ocurre en una alta prevalencia, sin embargo, se necesi- } \\
\text { tan más estudios. }\end{array}$ & $\begin{array}{l}\text { Estudio de } \\
\text { cohorte. }\end{array}$ \\
\hline $\begin{array}{l}\text { Nilsson et al. } \\
(2019)\end{array}$ & 31 pacientes. & $\begin{array}{l}\text { Apertura de espacio y posterior } \\
\text { instalación de implante oseoin- } \\
\text { tegrado. }\end{array}$ & $\begin{array}{l}\text { La mayoría de las coronas sobre implantes no mostra- } \\
\text { ron infraposición; un } 32 \% \text { mostró una leve infraposición } \\
\text { y sólo uno de los sujetos una franca infraposición. Ade- } \\
\text { más, no se encontró relación entre la edad y cuando } \\
\text { se instaló el implante, ni desplazamiento respecto a los } \\
\text { dientes vecinos. }\end{array}$ & $\begin{array}{l}\text { Estudio de } \\
\text { cohorte. }\end{array}$ \\
\hline
\end{tabular}


Tabla 1: Publicaciones incluidas, número de participantes, tratamiento evaluado, resultados y tipo de estudio. (continuación)

\begin{tabular}{|c|c|c|c|c|}
\hline $\begin{array}{l}\text { Melsen et al. } \\
(2015)\end{array}$ & $\begin{array}{l}4 \text { perros } \\
\text { Beagle. }\end{array}$ & $\begin{array}{l}\text { Apertura de espacio y pos- } \\
\text { terior instalación de implante } \\
\text { oseointegrado: Microtornillos } \\
\text { de ortodoncia. }\end{array}$ & $\begin{array}{l}\text { La instalación de un microtornillo, ayuda a que el hueso } \\
\text { aumente en densidad no solo en las áreas cercanas } \\
\text { a este, sino también en el sitio donde irá a futuro el } \\
\text { implante. }\end{array}$ & $\begin{array}{l}\text { Estudio compa- } \\
\text { rativo. }\end{array}$ \\
\hline $\begin{array}{l}\text { Plakwicz et al. } \\
\text { (2016) }\end{array}$ & 1 paciente. & $\begin{array}{l}\text { Autotrasplante dental, versus, } \\
\text { apertura de espacio y posterior } \\
\text { instalación de implante oseoin- } \\
\text { tegrado. }\end{array}$ & $\begin{array}{l}\text { Los tejidos periodontales alrededor del implante suelen } \\
\text { mostrar signos de recesión, mientras que los sitios } \\
\text { cercanos al diente trasplantado no muestran diferencias } \\
\text { respecto al diente natural. Se recomienda el autotras- } \\
\text { plante en el sector anterior, en caso de ser posible. }\end{array}$ & Reporte de caso. \\
\hline $\begin{array}{l}\text { Richardson et al. } \\
(2001)\end{array}$ & - & $\begin{array}{l}\text { Apertura de espacio y posterior } \\
\text { instalación de implante oseoin- } \\
\text { tegrado. }\end{array}$ & $\begin{array}{l}\text { Las raíces de los dientes vecinos al sector donde se } \\
\text { instalará el implante, deben ser paralelas o levemen- } \\
\text { te divergentes, con el fin de tener suficiente hueso } \\
\text { remanente y espacio adecuado entre implante y corona } \\
\text { protésica. } \\
\text { Se dice que es el tratamiento de elección, porque pre- } \\
\text { serva la estructura dentaria y el hueso, dando además } \\
\text { estética y función. }\end{array}$ & $\begin{array}{l}\text { Revisión narra- } \\
\text { tiva. }\end{array}$ \\
\hline $\begin{array}{l}\text { Sánchez et al. } \\
\text { (2012) }\end{array}$ & - & Autotrasplante dental. & $\begin{array}{l}\text { La literatura sugiere que los autotrasplantes pueden ser } \\
\text { una alternativa de sustitución a corto, mediano y largo } \\
\text { plazo de dientes perdidos, aunque el nivel de eviden- } \\
\text { cia es III, ya que los estudios revisados son de tipo } \\
\text { descriptivos. }\end{array}$ & $\begin{array}{l}\text { Revisión siste- } \\
\text { mática }\end{array}$ \\
\hline $\begin{array}{l}\text { Intra et al. } \\
(2014)\end{array}$ & 1 paciente. & Autotrasplante dental. & $\begin{array}{l}\text { El Autotrasplante dental es una buena alternativa en } \\
\text { comparación con los implantes en agenesia o trauma. } \\
\text { En el caso clínico, el autotrasplante sigue el crecimiento } \\
\text { normal de los procesos dentoalveolares y preserva la } \\
\text { función de los tejidos periodontales. }\end{array}$ & Reporte de caso \\
\hline $\begin{array}{l}\text { Priest et al. } \\
\text { (2019) }\end{array}$ & - & $\begin{array}{l}\text { Cierre de espacio con aparato- } \\
\text { logía fija de ortodoncia. } \\
\text { Rehabilitación con pónticos de } \\
\text { un ala. }\end{array}$ & $\begin{array}{l}\text { Ambos tratamientos presentan buenos resultados y } \\
\text { estética a lo largo del tiempo si son realizados de forma } \\
\text { correcta. La decisión debe basarse en la expectativa } \\
\text { del paciente, la clínica, costos asociados y el pronós- } \\
\text { tico. }\end{array}$ & $\begin{array}{l}\text { Revisión narra- } \\
\text { tiva. }\end{array}$ \\
\hline $\begin{array}{l}\text { Garnett et al. } \\
(2006)\end{array}$ & 45 pacientes. & $\begin{array}{l}\text { Rehabilitación con pónticos de } \\
\text { resina compuesta. }\end{array}$ & $\begin{array}{l}\text { De } 73 \text { puentes, } 30 \text { se descementaron al menos en una } \\
\text { ocasión }(41,1 \%), 6 \text { fueron por trauma }(20 \%) \text {. } \\
\text { El tiempo de supervivencia fue de } 59.3 \text { meses, con una } \\
\text { media de } 59 \text { meses. } \\
\text { En pacientes adultos la supervivencia fue mayor en } \\
\text { comparación con pacientes jóvenes. } \\
\text { El riesgo de falla fue } 3.9 \text { veces mayor en niños, mien- } \\
\text { tras que } 2.9 \text { veces en estudiantes. } \\
\text { La restauración con pónticos puede ser una opción } \\
\text { adecuada para el tratamiento definitivo. }\end{array}$ & $\begin{array}{l}\text { Estudio retros- } \\
\text { pectivo observa- } \\
\text { cional. }\end{array}$ \\
\hline $\begin{array}{l}\text { Tezulas et al. } \\
(2018)\end{array}$ & - & $\begin{array}{l}\text { Rehabilitación con puentes } \\
\text { cerámicos cementados con } \\
\text { resina. }\end{array}$ & $\begin{array}{l}\text { Es probable que los puentes de cerámica de un ala, } \\
\text { sean más exitosos que el diseño de puentes con dos } \\
\text { retenedores en la región anterior, sin embargo, la evi- } \\
\text { dencia es limitada. }\end{array}$ & $\begin{array}{l}\text { Revisión siste- } \\
\text { mática. }\end{array}$ \\
\hline $\begin{array}{l}\text { Stumbras et al. } \\
(2019)\end{array}$ & - & $\begin{array}{l}\text { Preservación de alveolos pos- } \\
\text { terior a extracción utilizando } \\
\text { materiales de injerto óseo y } \\
\text { concentración plaquetaria } \\
\text { autóloga. }\end{array}$ & $\begin{array}{l}\text { A pesar de la existencia de múltiples materiales para la } \\
\text { preservación alveolar, ninguno de ellos detiene comple- } \\
\text { tamente la reabsorción ósea posterior a la extracción } \\
\text { de un diente. }\end{array}$ & $\begin{array}{l}\text { Revisión siste- } \\
\text { mática. }\end{array}$ \\
\hline $\begin{array}{l}\text { Avila et al. } \\
(2012)\end{array}$ & 1 paciente. & $\begin{array}{l}\text { Apertura de espacio e insta- } \\
\text { lación de implante oseointe- } \\
\text { grado. }\end{array}$ & $\begin{array}{l}\text { Los implantes deben ser la primera opción de tra- } \\
\text { tamiento conservador para la agenesia de incisivos } \\
\text { laterales superiores. } \\
\text { La decisión debe ser multidisciplinaria, debido a que el } \\
\text { éxito depende de seguir las etapas adecuadas. }\end{array}$ & Reporte de caso. \\
\hline
\end{tabular}


Tabla 1: Publicaciones incluidas, número de participantes, tratamiento evaluado, resultados y tipo de estudio. (continuación)

\begin{tabular}{|c|c|c|c|c|}
\hline $\begin{array}{l}\text { Andersson et al. } \\
(2013)\end{array}$ & 57 pacientes. & $\begin{array}{l}\text { Apertura de espacio y posterior } \\
\text { instalación de implante oseoin- } \\
\text { tegrado. }\end{array}$ & $\begin{array}{l}\text { De un total de } 47 \text { pacientes, } 2 \text { implantes fallaron, y se } \\
\text { reemplazaron } 8 \text { restauraciones realizadas con prótesis } \\
\text { fija unitaria. } 3 \text { de las coronas reemplazadas fueron por } \\
\text { infraposición. Alrededor del } 40 \% \text { de los pacientes mos- } \\
\text { traron signos de infraposición de la corona, de manera } \\
\text { similar en pacientes jóvenes y adultos, pero fue más } \\
\text { frecuentemente observado en mujeres. Los pacientes } \\
\text { se mostraron más satisfechos con los resultados que } \\
\text { los clínicos que participaron del estudio. }\end{array}$ & $\begin{array}{l}\text { Estudio prospec- } \\
\text { tivo observacio- } \\
\text { nal. }\end{array}$ \\
\hline $\begin{array}{l}\text { Zarone et al. } \\
(2006)\end{array}$ & 30 pacientes. & $\begin{array}{l}\text { Apertura de espacio y posterior } \\
\text { instalación de implante oseoin- } \\
\text { tegrado. }\end{array}$ & $\begin{array}{l}\text { Se muestran valores satisfactorios de reabsorción } \\
\text { marginal y buenas condiciones del tejido periimplantario } \\
\text { alrededor de los implantes. }\end{array}$ & Ensayo clínico. \\
\hline $\begin{array}{l}\text { Branzen et al. } \\
(2015)\end{array}$ & 36 pacientes. & $\begin{array}{l}\text { Apertura de espacio y posterior } \\
\text { instalación de implante oseoin- } \\
\text { tegrado. }\end{array}$ & $\begin{array}{l}\text { Desde la perspectiva del paciente, la mayoría estaba } \\
\text { satisfecho en términos de función y estética. Un tercio } \\
\text { de los pacientes quiso el cambio de las coronas sobre } \\
\text { implantes, por una alteración del tejido blando, lo que } \\
\text { se consideró necesario. }\end{array}$ & $\begin{array}{l}\text { Estudio retros- } \\
\text { pectivo observa- } \\
\text { cional. }\end{array}$ \\
\hline $\begin{array}{l}\text { Silveira et al. } \\
(2016)\end{array}$ & - & $\begin{array}{l}\text { Apertura de espacio y pos- } \\
\text { terior instalación de implante } \\
\text { oseointegrado, versus, cierre } \\
\text { de espacio con aparatología } \\
\text { fija de ortodoncia. }\end{array}$ & $\begin{array}{l}\text { Las coronas sobre implantes muestran peores índices } \\
\text { periodontales en relación al cierre de espacios por } \\
\text { ortodoncia; en términos estéticos, las coronas sobre im- } \\
\text { plantes muestran una mayor crítica en personas comu- } \\
\text { nes, pacientes y dentistas; La presencia o ausencia de } \\
\text { un cambio de posición en el canino, no mostró cambios } \\
\text { en la función oclusal, ni signos o síntomas de TTM. }\end{array}$ & $\begin{array}{l}\text { Revisión narra- } \\
\text { tiva. }\end{array}$ \\
\hline $\begin{array}{l}\text { Dierens et al. } \\
(2013)\end{array}$ & 21 pacientes. & $\begin{array}{l}\text { Apertura de espacio y posterior } \\
\text { instalación de implante oseoin- } \\
\text { tegrado. }\end{array}$ & $\begin{array}{l}\text { Los tejidos blandos alrededor del implante se mantu- } \\
\text { vieron estables en pacientes con salud periodontal. Los } \\
\text { dientes vecinos, mostraron recesiones y erupciones, } \\
\text { respecto a la línea media facial. } \\
\text { En cuanto a parámetros estéticos, se calificó como } \\
\text { pobre. }\end{array}$ & $\begin{array}{l}\text { Estudio retros- } \\
\text { pectivo observa- } \\
\text { cional. }\end{array}$ \\
\hline $\begin{array}{l}\text { Rafalowicz et al. } \\
\text { (2019) }\end{array}$ & 129 pacientes. & $\begin{array}{l}\text { Apertura de espacio y posterior } \\
\text { instalación de implante oseoin- } \\
\text { tegrado; Rehabilitación con } \\
\text { pónticos; Cierre de espacio } \\
\text { mediante aparatología fija de } \\
\text { ortodoncia. }\end{array}$ & $\begin{array}{l}\text { Se concluye que el uso de microtornillos de ortodoncia, } \\
\text { la rehabilitación de implantes oseointegrados y el uso } \\
\text { de dentaduras parciales, son los tratamientos más } \\
\text { efectivos. } \\
\text { El cambiar la forma del canino, provocó cambios de } \\
\text { aspecto clínico aceptables en dos tercios de los casos. }\end{array}$ & $\begin{array}{l}\text { Estudio retros- } \\
\text { pectivo observa- } \\
\text { cional. }\end{array}$ \\
\hline $\begin{array}{l}\text { Amm et al. } \\
(2019)\end{array}$ & 30 pacientes. & $\begin{array}{l}\text { Cierre de espacio con aparato- } \\
\text { logía fija de ortodoncia asistido } \\
\text { con anclaje esqueletal. }\end{array}$ & $\begin{array}{l}\text { El cierre de espacio con aparatología fija de ortodoncia } \\
\text { en pacientes clase II y clase III esqueletal, utilizando } \\
\text { anclajes esqueletales intraorales en conjunto con elásti- } \\
\text { cos clase III permite buenos resultados clínicos. } \\
\text { Se establece una adecuada oclusión mesializando el } \\
\text { canino, como también una resolución de la discrepan- } \\
\text { cia intermaxilar. } \\
\text { El perfil de los tejidos blandos se mantiene armonioso. } \\
\text { La estética de la sonrisa también mejoró después del } \\
\text { tratamiento de ortodoncia. }\end{array}$ & $\begin{array}{l}\text { Estudio de } \\
\text { cohorte. }\end{array}$ \\
\hline $\begin{array}{l}\text { Stevnik et al. } \\
(2006)\end{array}$ & - & $\begin{array}{l}\text { Autotrasplante dental y cierre } \\
\text { de espacio con aparatología } \\
\text { fija de ortodoncia. }\end{array}$ & $\begin{array}{l}\text { El autotrasplante dental ha demostrado tasas de } \\
\text { sobrevivencia a largo plazo de más de un } 90 \% \text {. En } \\
\text { comparación con los incisivos laterales naturales, el au- } \\
\text { totrasplante se rodea de un tejido blando con caracte- } \\
\text { rísticas similares. El resultado estético fue satisfactorio, } \\
\text { particularmente cuando el diente autotrasplantado fue } \\
\text { alineado con aparatos fijos de ortodoncia. } \\
\text { Cuando los pacientes se mostraban descontentos, la } \\
\text { razón fue por una apariencia subóptima de la recons- } \\
\text { trucción con resina. } \\
\text { El mejor beneficio de reemplazar el diente ausente por } \\
\text { un sucesor biológico, ya sea con cierre de espacio y } \\
\text { mesialización del canino, o autotrasplante dental, es } \\
\text { que existe una compatibilidad biológica y permanencia } \\
\text { de los resultados clínicos. }\end{array}$ & $\begin{array}{l}\text { Revisión narra- } \\
\text { tiva. }\end{array}$ \\
\hline
\end{tabular}




\section{Bibliografía}

1. Schneider UEM, Moser L. Substituting congenitally missing maxillary lateral incisors with implant-borne crowns: A topic still worth a discussion? Semin Orthod. 2020;26(1):33-51.

2. Schneider UEM, Moser L, Pellitteri G, Siciliani G. Orthodontic space closure vs. implant-borne crowns in patients with congenitally missing maxillary lateral incisors. $\mathrm{J}$ Clin Orthod. 2018;(5):13.

3. Cocconi R, Rapa S. Unilateral agenesis of the maxillary lateral incisor: space closure versus space preservation in growing patients. Semin Orthod. 2020;26(1):2432.

4. Cope JB, McFadden D. Temporary replacement of missing maxillary lateral incisors with orthodontic miniscrew implants in growing patients: rationale, clinical technique, and long-term results. J Orthod. 2014;41(1):62-74.

5. Antonarakis GS, Prevezanos P, Gavric J, Christou P. Agenesis of maxillary lateral incisor and tooth replacement: cost-effectiveness of different treatment alternatives. Int J Prosthodont. 2014;27(3):257-63.

6 . Rosa M. Missing teeth in the smile area: space closure in all malocclusions looking for long term health, esthetics and function. Semin Orthod. 2020;26(1):52-60.

7. Vilhjálmsson VH, Knudsen GC, Grung B, Bårdsen A. Dental auto-transplantation to anterior maxillary sites. Dent Traumatol. 2011;27(1):23-9.

8. Priest $\mathrm{G}$. The treatment dilemma of missing maxillary lateral incisors-Part II: Implant restoration. J Esthet Restor Dent. 2019;31(4):319-26.

9. Kavadia S, Papadiochou S, Papadiochos I, Zafiriadis L. Agenesis of maxillary lateral incisors: a global overview of the clinical problem. Orthod Art Pract Dentofac Enhanc. 2011;12(4):296-317.

10. Kiliaridis S, Sidira M, Kirmanidou $\mathrm{Y}$, Michalakis $\mathrm{K}$. Treatment options for congenitally missing lateral incisors. Eur J Oral Implantol. 2016;9(1):5-24.

11. Park JH, Okadakage S, Sato $\mathrm{Y}$, Akamatsu $\mathrm{Y}$, Tai K. Orthodontic treatment of a congenitally missing maxillary lateral incisor: Congenitally missing maxillary lateral treatment. J Esthet Restor Dent. 2010;22(5):297-312.

12. Wagner L, Rafałowicz B. Remodeling of the maxillary canine to the shape of the lateral incisor as a method of hypodontia treatment - 10-year clinical observations. J Stomatol. 2019;72(1):13-6.

13. Thilander B. Orthodontic space closure versus implant placement in subjects with missing teeth. J Oral Rehabil. 2008;35(1):64-71.

14. Bernard JP, Schatz JP, Christou P, Belser U, Kiliaridis S. Long-term vertical changes of the anterior maxillary teeth adjacent to single implants in young and mature adults. A retrospective study. J Clin Periodontol. 2004;31(11):1024-8.

15. Cocchetto R, Pradies G, Celletti R, Canullo L. Continuous craniofacial growth in adult patients treated with dental implants in the anterior maxilla. Clin Implant Dent Relat Res. 2019;21(4):627-34.

16. Otaño, R. Crecimiento y desarrollo craneofacial [Internet]. [citado 30/10/2020] Disponible en: http://articulos.sld.cu/ortodoncia/files/2009/12/crec-y-des-preg.pdf

17. Fernández J. Como y hasta cuando crece la cara de nuestro paciente. [Internet]. [citado 02/01/2021]. Disponible en: http://ortoface.com/wp-content/uploads/2016/06/ Como-y-hasta-cuando-crece-la-cara-de-nuestro-paciente.pdf

18. Nilsson A, Johansson L-Å, Stenport VF, Wennerberg A, Ekfeldt A. Infraposition of anterior maxillary implant-supported single-tooth restorations in adolescent and adult patients-A prospective follow-up study up to 6 years. Clin Implant Dent Relat Res. 2019;21(5):953-9.

19. Melsen B, Huja SS, Chien H-H, Dalstra M. Alveolar bone preservation subsequent to miniscrew implant placement in a canine model. Orthod Craniofac Res. 2015;18(2):77-85.

20. Plakwicz P, Fudalej P, Czochrowska EM. Transplant vs implant in a patient with agenesis of both maxillary lateral incisors: A 9-year follow-up. Am J Orthod Dentofac Orthop. 2016;149(5):751-6.

21. Richardson G, Russell KA. Congenitally missing maxillary lateral incisors and orthodontic treatment considerations for the single-tooth implant. J Can Dent Assoc. $2001 ; 67(1): 25-8$.

22. Sánchez SB, Cardozo LA, Ruiz LMR. Autotrasplantes dentales: revisión sistemática de la literatura. Univ Odontológica. 2012;31(66):133-43.

23. Intra JBG, Roldi A, Brandão RCB, de Araújo Estrela CR, Estrela C. Autogenous premolar transplantation into artificial socket in maxillary lateral incisor site. J Endod. 2014:40(11):1885-90.

24. Priest G. The treatment dilemma of missing maxillary lateral incisors-Part I: Canine substitution and resin-bonded fixed dental prostheses. J Esthet Restor Dent. 2019;31(4):311-8.

25. Garnett MJ, Wassell RW, Jepson NJ, Nohl FS. Survival of resin-bonded bridgework provided for post-orthodontic hypodontia patients with missing maxillary lateral incisors. Br Dent J. 2006;201(8):527-34

26. Tezulas E, Yildiz C, Evren B, Ozkan Y. Clinical procedures, designs, and survival rates of all-ceramic resin-bonded fixed dental prostheses in the anterior region: A systematic review. J Esthet Restor Dent. 2018;30(4):307-18.

27. Ferraris MEG de, Campos Muñoz A. Histología y embriología bucodental. 2a Ed. Santiago, Chile: Editorial Médica Panamericana;2002.

28. Stumbras A, Kuliesius P, Januzis G, Juodzbalys G. Alveolar ridge preservation after tooth extraction using different bone graft materials and autologous platelet concentrates: a systematic review. J Oral Maxillofac Res. 2019;10(1):2-11.

29. Avila E, De Molon R, Molo F, Barros L, Filho L, Cardoso M, et al. Multidisciplinary approach for the aesthetic treatment of maxillary lateral incisors agenesis: Thinking about implants? Oral Surg Oral Med Oral Pathol Oral Radiol. 2012;114(5):22-28.

30. Andersson B, Bergenblock S, Fürst B, Jemt T. Long-Term function of singleimplant restorations: a 17- to 19-year follow-up study on implant infraposition related to the shape of the face and patients' satisfaction. Clin Implant Dent Relat Res. 2013:15(4):471-80.

31. Zarone F, Sorrentino R, Vaccaro F, Russo S. Prosthetic treatment of maxillary lateral incisor agenesis with osseointegrated implants: a 24-39-month prospective clinical study. Clin Oral Implants Res. 2006;17(1):94-101.

32. Branzén M, Eliasson A, Arnrup K, Bazargani F. Implant-Supported single crowns replacing congenitally missing maxillary lateral incisors: a 5-year follow-up. Clin Implant Dent Relat Res. 2015;17(6):1134-40.

33. Silveira GS, de Almeida NV, Pereira DMT, Mattos CT, Mucha JN. Prosthetic replacement vs space closure for maxillary lateral incisor agenesis: A systematic review. Am J Orthod Dentofac Orthop. 2016;150(2):228-37.

34. Dierens M, de Bruecker E, Vandeweghe S, Kisch J, de Bruyn H, Cosyn J. Alterations in soft tissue levels and aesthetics over a 16-22 year period following single implant treatment in periodontally-healthy patients: a retrospective case series. J Clin Periodontol. 2013;40(3):311-8.

35. Rafałowicz B, Wagner L. Assessment of hypodontia treatment of maxillary lateral incisors in adult patients after 9 years of follow-up: a retrospective study. Int $J$ Prosthodont. 2019;32(1):9-13

36. Amm EW, Antoszewska-Smith J, Boley J. Canine substitution of congenitally missing maxillary lateral incisors in Class I and Class III malocclusions by using skeletal anchorage. Am J Orthod Dentofacial Orthop. 2019;156(4):512-521.

37. Stenvik A, Zachrisson BU. Missing anterior teeth: orthodontic closure and transplantation as viable options to conventional replacements. Endod Top. 2006;14(1):41-50. 